\title{
THE DESIGN OF THE RESEARCH METHOD IN GRADUATE RESEARCH WORK
}

\author{
Imane Ghazlane $^{1,2}$, Bouzekri Touri ${ }^{1}$, Mohamed Bergadi ${ }^{1}, \&$ Khalid Marnoufi $^{1}$ \\ ${ }^{1}$ Multidisciplinary Laboratory of Information, Communication and Education Sciences and Technology \\ (LAPSTICE), Ben M'sik Faculty of Science, Hassan II University, post box 795, Casablanca (Morocco) \\ ${ }^{2}$ Higher Institute of Nursing and Health Technology Professions Casablanca-Settat, Morocco Street \\ Faidouzi Mohamed.20250 Casablanca (Morocco)
}

\begin{abstract}
Regardless of the discipline or institution in which the scientific research was carried out, the "method" section is present. However, many research works lack relevance and suffer from methodological problems. However, method remains the foundation of all research work that can inevitably influence results that can affect problem solving, national development, and threaten our quality of life in general.

This work is an exploratory study of research methods used in graduation projects in the following disciplines (health sciences, engineering, biological and agronomic sciences, economic and social sciences).

The method used in this work is based on three investigative tools: (a) comparison of national scientific production according to a few references (b) semi-structured survey by interviewing supervisors of final dissertations and theses in different selected disciplines (b) systematic analysis of fifty final dissertations. The works was obtained from the libraries of the University Hassan II of Casablanca in different disciplines, submitted between 2014 and 2018. The method part was analyzed, taking into account the key processes and concepts of each discipline, to highlight the elements of the research method, namely the type of research, sampling, procedure or conduct of research, mode of data collection, data analysis, results, and discussion.

Among the key findings of this study, (a) a decline in national scientific output compared to previous years and (b) shortcomings in research design. Our contribution lies in remedying the standardization of the method and adapting it to the contexts of the needs of different disciplines.
\end{abstract}

Keywords: Scientific research, research method, dissertation work.

\section{Introduction}

Some factors influence scientific judgment and decision-making, and can bias and corrupt research (Steneck 2004; Shamoo and Resnik 2015), so many strategies are developed to promote scientific integrity, which include the "integrity of results" and "integrity of process».

The «integrity of outcomes" is concerned the achievement of goals and the production of results that are empirically adequate; and integrity of process is the commitments to the standards that promote those goals (Shamoo and Resnik 2015). However, this scientific integrity can be at risk throughout the research process.

Regarding the method, it is one of the sections of the research process and contains sample, type of study, instruments, procedure, data analysis... (Fortin, 2010).

Furthermore, from a methodological point of view, if such subsections are not applied accurately, and if they do not follow the standards, they can lead to empirically inadequate results (Shamoo and Resnik, 2015).

In addition, when the researcher has used inappropriate method to obtain his or her results, and if he or she does not follow the standards, he or she may violate scientific integrity. (Douglas, 2014).

Several researchers have agreed that methodological integrity in research is a priority; it is an approach that must be applied to all areas of scientific research in order to produce empirically adequate conclusions.

Moreover, the lack of prerequisites in the conduct of research is at the root of the difficulties in publishing quality papers, and the delay in promotion of many African researchers (Firman and all, 2015).

For this, the research method must be adjusted and based on the norms and standards, to reach the research objectives and to provide useful results and conclusions to serve societal sectors. 
The purpose of this study is to explore the research design on the empirical studies of the five disciplines (engineering sciences, health sciences, biological and agricultural sciences, economic and social sciences) of the scientific production of the University HASSAN II DU CASABLANCA in order to compare and contrast them with the norms and standards.

\section{Method}

\subsection{National scientific production according to a few references}

The indicator number of theses introduced as a performance indicator in the development contract 'State University of the Emergency Program'. However, at the national level, the number of doctoral students is accumulating, without the same graduation rate. In 2014, the number of students reached nearly 18600 while the number of doctorates supported was less than 1200, with a graduation percentage of 6.4. This rate is relatively low compared to Tunisia, which is $7.2 \%$, South Africa 12.6 and France 19.3.

In addition, the number of doctoral degrees awarded, all disciplines combined, is very low (676 in 2009) and (392 in 2018) (sources: CRUH2C AND Statistical direction).

There are several reasons related to the system and/or process that constrain research in Morocco. The Higher Council of Education, Training and Scientific Research (2017) indicated that $60 \%$ of PhD students have not received training in bibliographical research and there is any access to knowledge without methodological preparation and an initiation to bibliographical research. However, no study is interested on research design.

\subsection{The interview survey}

The interview involved teacher-researchers supervising theses and dissertations work, all the interviews was recorded in audio to be processed on "Nvivo" after having received their consent through an explanation of purpose and orientation of the study.

\subsection{Analysis of dissertation work}

Fifty theses were obtained from the libraries of Hassan II University; we included engineering sciences; health sciences, biology and agriculture, social and economic sciences.

The conceptual framework is the design of the research, which includes the type of study, the sample, the instruments, the data collection, the data analysis, the results and the discussion.

The triangulation of the three survey methods, which involves the collection of a few indicators at the doctoral level, the semi-structured interview of teacher-researchers and the analysis of the research work of master's students, was undertaken in order to compare and reinforce the results and objective of the study.

\section{Result}

\subsection{Interview results}

A textual analysis of the interviews with "Nvivo" revealed some repeated concepts. And a "Method" is the predominant factor affecting the students' research work. Furthermore, the data analysis and collection is the one component that can threat the integrity of the research method in research work of students.

\subsection{Results of the research analysis}

The results of the study showed that in medicine: the type of study and ethical considerations were indicated in all work. $60 \%$ of the studies emphasized the number and characteristics of the sample and only $40 \%$ mentioned their sampling techniques, however, the fidelity and validity of the data collection instruments were highlighted in only $40 \%$ of the studies. In addition, all studies described their procedure, their statistical analysis and their results. However, the discussion section considers the other writings in only $40 \%$ of the cases, and the conclusion section appears in only $40 \%$ of the works.

In biology and agriculture, just $30 \%$ of the studies declared the type of study, the predominant criterion in the sampling section is the study environment in $100 \%$ of the cases. In addition, the sampling technique, the number and characteristics of the sample appear in only $40 \%$ of the studies, and the precision and validity of the measuring instruments were not explicit in $40 \%$ of the cases. Moreover, just $40 \%$ explain their significance level; however, in the discussion section all studies indicate that the results are consistent with other publications through comparative studies.

In sociology, the type of study appears in only $10 \%$ of the cases. The study environment and the number and characteristics of the sample appear in $60 \%$ of the cases. $70 \%$ of the writings create their instruments, however, their validity was not emphasized. The statistical analysis reveals the results in all of studies, and only $10 \%$ of the cases carry out a discussion part. 
In economics, $40 \%$ of the studies indicate their type of study, and the study environment is present in all of them. However, $50 \%$ indicate the characteristics of sample, furthermore, $80 \%$ use instruments created for the purpose of the study and just $10 \%$ clarified their validity, and only $30 \%$ of the cases described their procedure in detail, $40 \%$ pronounced their significance of the results. Moreover, $20 \%$ of the works used other literature in the discussion, and just $30 \%$ cited their study limitations.

In engineering sciences, the total of work mentions their study environment, but the type of study is not reported in all of paper. The instruments are all imported with their validities, while $20 \%$ have not given results; by the way, $30 \%$ of the studies reported their study limitations.

\section{Discussion}

This survey is necessary because it highlights the practice of research method, which is described as the guarantor of methodological rigor, as it contains subsections, and because they influence each other.

This diagnosis makes it possible to raise the gaps in the practice of the research method for improvement purposes.

Given the results of the interviews with teacher-researchers, it was found that the sections most at stake are data analysis and data collection. In addition, analysis of research works indicates the deficiency in all method sections.

Because, most studies do not describe their procedures for either data collection or data analysis, which limits their transparency (Fortin, 2016). Moreover, the statistical analysis is the main component that allows research to be empirical rather than abstract, it allows us to confirm our findings, furthermore, Assar (2015) stipulate that the artifact must meet the needs of the applicant, and it must be adapted to the context.

\section{Conclusion}

The aim of this study is diagnosis of the application of the research method by students in different disciplines, and, the results obtained show that, the rules and standards relating to the normative practices of the method are not respected in most of the works.

In order to remedy the standardization of the research method, and its adaptation to the contexts of the needs of different disciplines, there are some recommendations:

- The reinforcement of research methodology courses within training courses (bachelor's degrees, master's degrees, and doctorate) in all disciplines.

- The requirement for the development of a research work plan and the clarification of the method

- The characterization of each method section and linking all parts of the method in order to respect the epistemological act

- Teaching based on the universal standards of critical thinking to generate critical thinkers.

- At the strategic level, embedding the research culture and adherence to norms and standards to ensure the integrity of the research method

- The choice and/or design of data collection and analysis instruments must be justified, rational and clarified.

\section{References}

Assar, S (2015) Empirical research method in IS engineering, principles and applications. CRUH2C AND the Directorate of Statistics. DOI: 10.3166/isi.20.6.11-33

Douglas, H. (2014). Scientific integrity in a politicized world. https://www.researchgate.net/publication/281749386

Firmin, B and all. (2015). The research protocol: an indispensable step in the research process guaranteeing the validity of the results. DOI: $10.4267 / 2042 / 56336$

Fortin, F.M. (2010). Fondements et étapes du processus de recherche. Chenelière education.

Higher Council for Education, Training and Scientific Research. (2017).

Luc Van Campenhoudt, Jacques Marquet, Raymond Quivy (2017). Social Science Research Manual.

Manille. (2003), Methodology for Health Research. Guide de la formation aux méthodes de la recherche scientifique.

National Strategy for Scientific Research to 2025.

Shamoo, A. and D. Resnik. (2015). Responsible Conduct of Research, 3rd Ed. New York, NY: Oxford University Press. ISBN 978-0-19-937602-5

Steneck, N. (2004). ORI Introduction to Responsible Conduct of Research. Washington, DC: Office of Research Integrity. 